\title{
DÉCOUVERTE D'UN MURUS GALLICUS A LEVROUX (Indre)
}

\author{
par Olivier BUCHSENSCHUTZ et Ian B.M. RALSTON
}

Les travaux de recherche entrepris à Levroux depuis $1968^{1}$ nous ont amenés à effectuer un sauvetage, puis une fouille, sur le rempart qui entoure l'oppidum de la Colline des Tours (fig. 1). Sous le nom de "Bonnan" ou "Bonnens», cette station est connue depuis les prospections de l'archiviste A. Lemaigre ${ }^{2}$ dans les années $18 ; 0$ publiées par A. Blanchet ${ }^{3}$ et reprises par J. Wéchelette dans son Manuel : la découverte de milliers de monnaies et de plusieurs objets en bronze décorés laissait en effet supposer l'existence d'une importante agglomération de la Tène III sur cette colline.

La disposition rayonnante des parcelles, plantées de vignes et de vergers, le tracé des chemins et des talus qui encerclent la colline, suggéraient même la présence d'un rempart (fig. 2); mais il n'était pas possible d'affirmer qu'il ne s'agrissait pas "d'un simple jeu de la nature $»^{6}$, car en aucun endroit n'apparaissaient de traces évidentes de fortification. Ce sont finalement des anomalies géologiques observées au cours d'un sauvetage qui nous ont conduits à décourrir un murus gallicus enfoui à $2 \mathrm{~m}$ de profondeur.

Un sondage pratiqué sur le terrain où .J. Triomphe allait construire une maison (fig. 3) a mis au jour en juillet 1971 une couche composée de petits blocs de calcaire épaisse par endroits de plus d'un m. Le matériau a été rapporté de la plaine de Champagne qui commence au pied de la colline, à $300 \mathrm{~m}$ du sondage. Deux tranchées pratiquées en 1971 ont montré que cette couche a un pendage contraire à la pente actuelle du terrain : elle vient buter, en effet, sur un éboulis de pierres sèches, puis reprend $50 \mathrm{~cm}$ plus haut, formant comme une marche à contre-pente (fig. 1).

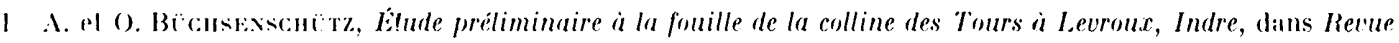
archéologique du Cientre, $\mathrm{X}, 1971, \mathrm{p} .2010 \mathrm{x}-2 \cdot 25$.

Association pour la defense al libludi du canton de Levroux, Recherches sur le Canton de Levroux (Indre), 1972, p. 103-112, sondiage 1.

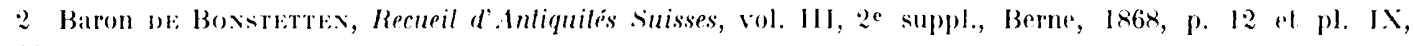
fi. $.9-11$.

3 1. Buxchet, Trailé des monnuies grauloises, Paris, 1905, passim.

4 J. DE.chliterte, Manuel..., vol. II, 3, Paris, 191.4, p. 969, 1456.

5) J. V. S. MEgiaw, Les fra!ments de feuilles de bronze décorées de Levrour, dans (iallia, XXVI, 1964, p. 33-41.

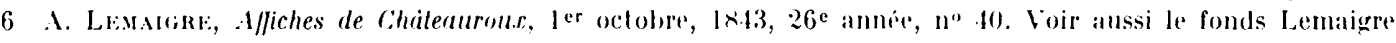
aux Archives de Chateauroux, dépouille par A. Chaslagnol, Revue de latcademie du Centre, 1955, p. 40-53. 


\section{Illustration non autorisée à la diffusion}
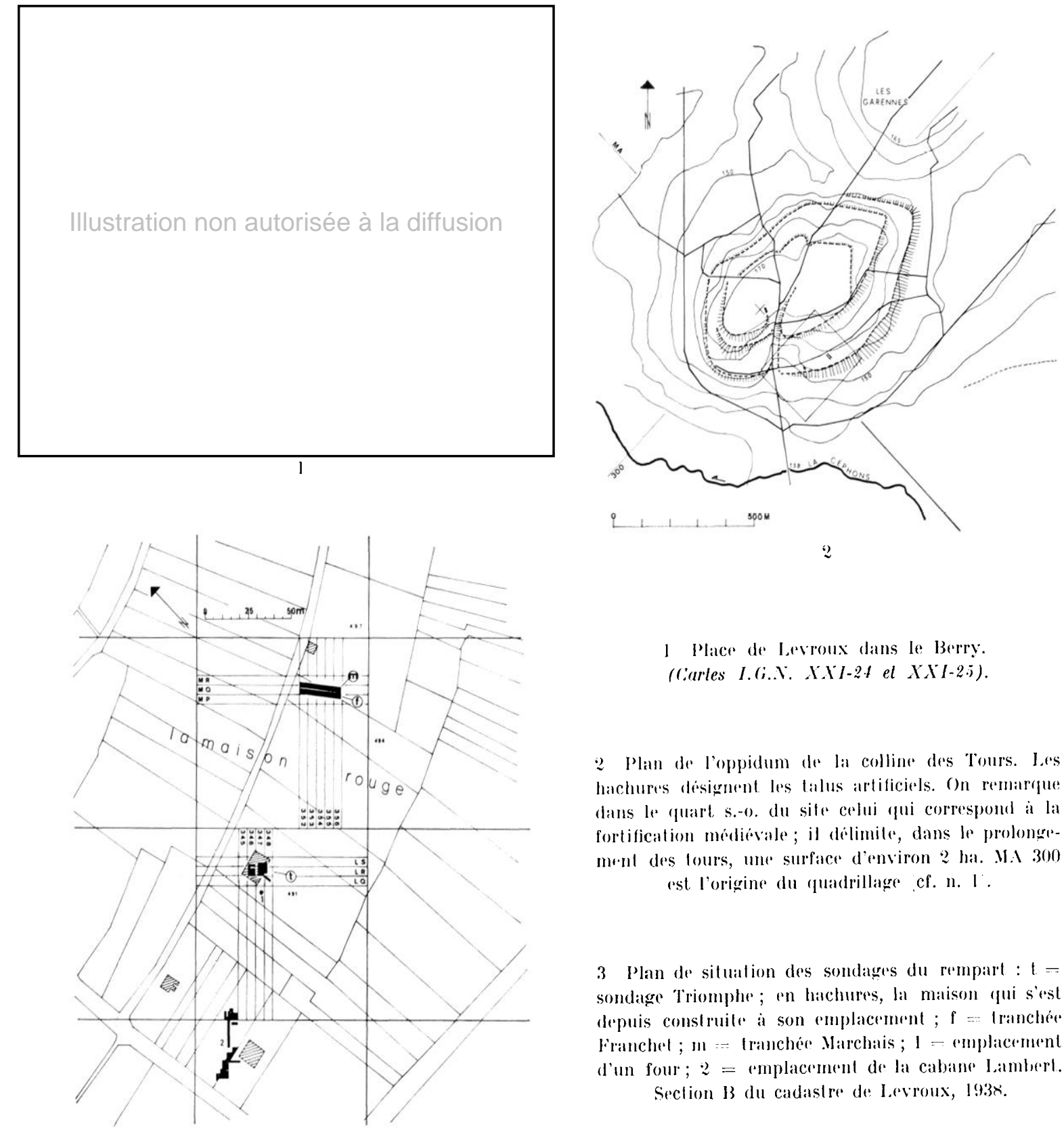

I Place de levroux dans le Berry

(Carles I.(i..). XXI-2.t el XXI-2.j).

2 Plan de l'oppidum de la colline des Tours. Les hachures designent les talus artificiels. On remarque dans le quart s.-o. du site celui qui correspond a la fortification modievale; il delimite, dans le prolongemenl des tours, une surface d'environ ? ha. .M. 300 est l'origine du quadrillage ef. n. I.

3 Plan de situalion des sondages du rempart : 1 = sondage Triomphe; en hachures, la maison qui s'est depuis conslruile a son emplacement ; f =.. Iranchee Franchel; $n$ :- lraucher Marchais ; 1 - emplacement d'un four ; $2=$ emplacement de la cabane Lambert. Section $B$ du cadastre de l.evroux, 1938 .

La présence exclusive de tessons du ier siècle nous a encouragés à poursuivre ce sondage pour trouver l'explication de cette anomalie. Sous avons alors découvert à clous en fer de section carrée, longs de 2j à $30 \mathrm{~cm}$, éléments caractéristiques du murus gallicus. L'exiguïté du sondage ne nous a cependant pas permis de comprendre à quelle partie du rempart nous avions affaire. Aussi avons-nous décillé d'ouvrir à la pelle mécanique? une tranchée longue de 25 , large de 2,50 et profonde de $2 \mathrm{~m}$ (tranchée $\mathrm{m}$ ) pour étudier

7 Nous remercions très vivement M. R. Viano qui a, comme toutes les fois où nous l'avons sollicité, effeclué gracierusement la tranchée à la pelle mécanique. 


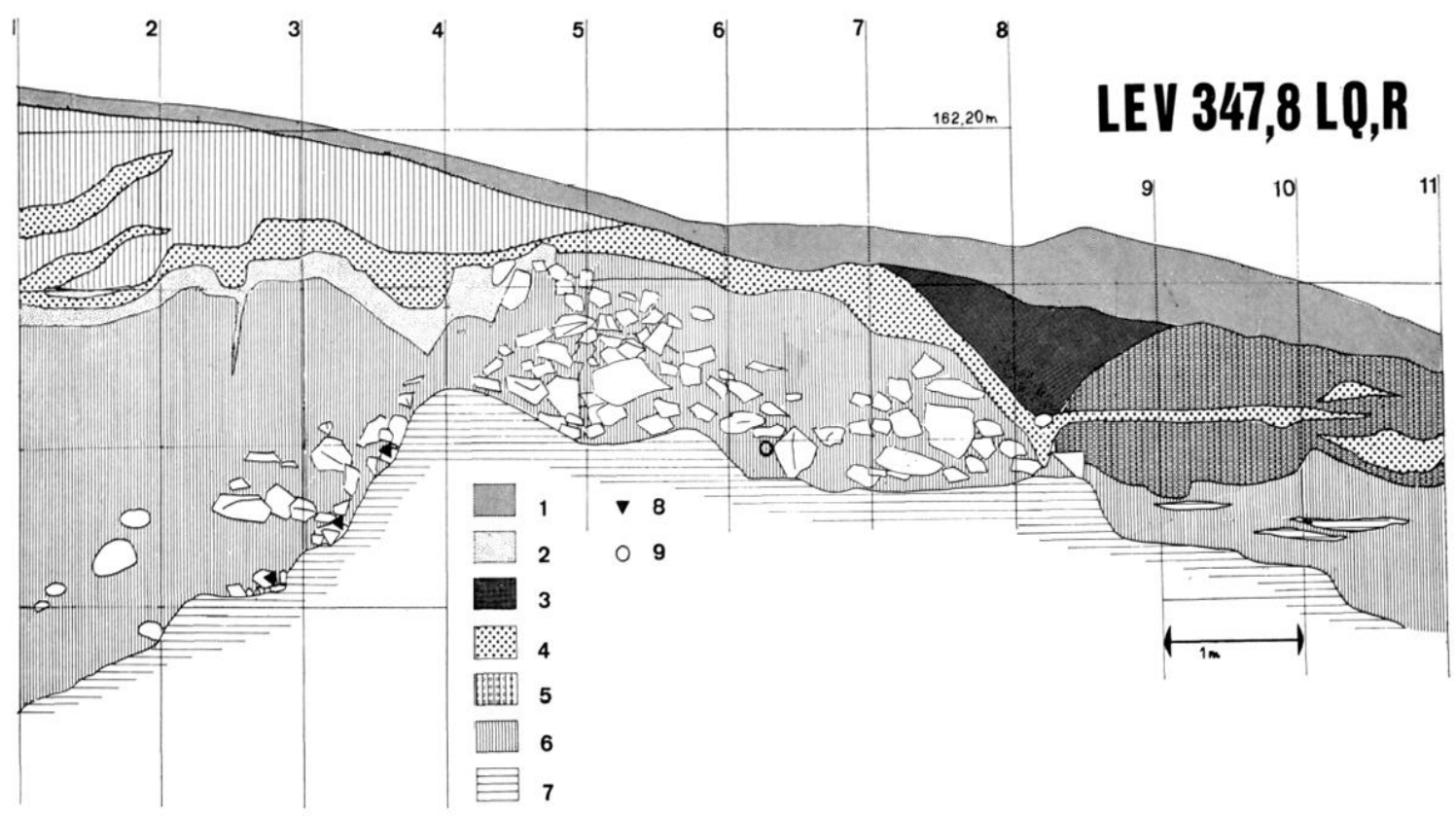

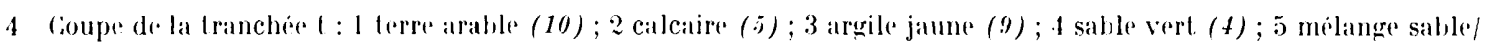
calcaire (4:-j) ; 6 argile grise marbrée rouge (2); 7 argile et sable de base (1); $x$ clous du murus gallicas ; 9 bracelel 3471.12090 .

le rempart dans son ensemble et fouiller les couches les plus profondes (fig. 5) ; une seconde tranchée (tranchée f) parallèle à la première a ensuite été fouillée à la main de la surface du sol à $1,50 \mathrm{~m}$ de profondeur pour étudier les couches superficielles : la chape de calcaire, le sommet du murus gallicus, l'éboulis du parement externe, et une couche d'occupation qui s'étend au sud du rempart (fig. 6).

\section{LA STRATIGRAPHIE}

La couche de surface (10) est peu épaisse et paurre en objets. Une carrière moderne a été creusée à l'extrémilé sud de la Iranchée f pour récupérer de la pierre.

On rencontre ensuite dans les 3 sondages un ensemble de couches alternées de sable et de calcaire épais de $2 \mathrm{~m}$. Ces couches ont un léger pendage vers le nord, alors que la pente actuelle du terrain descend vers le sud. Eilles sont coupées par trois failles, localisées sur la coupe dans les $2 \mathrm{e}, 4 \mathrm{e}$ et $13 \mathrm{e} \mathrm{m}$; dans tous les cas, c'est le compartiment nord qui s'est afiaaissé par rapport au compartiment sud, l'ensemble formant un "escalier". Le sommet des plans de faille est incliné vers le sud.

On peut distinguer dans la couche calcaire (j) deux horizons principaux : le premier apparail dans les compartiments $A$ et B, le second dans les compartiments B, C et I). Celte couche est presque stérile; elle n'a livré aucun objet qui ne puisse ètre altribué au $-1^{\text {er }}$ siecle.

L'ensemble complexe des horizons qui forment la couche sableuse verle (4) a livré un nombre de tessons très réduit, mais présentant une répartition des différents types analogue à celle des couches inférieures. 


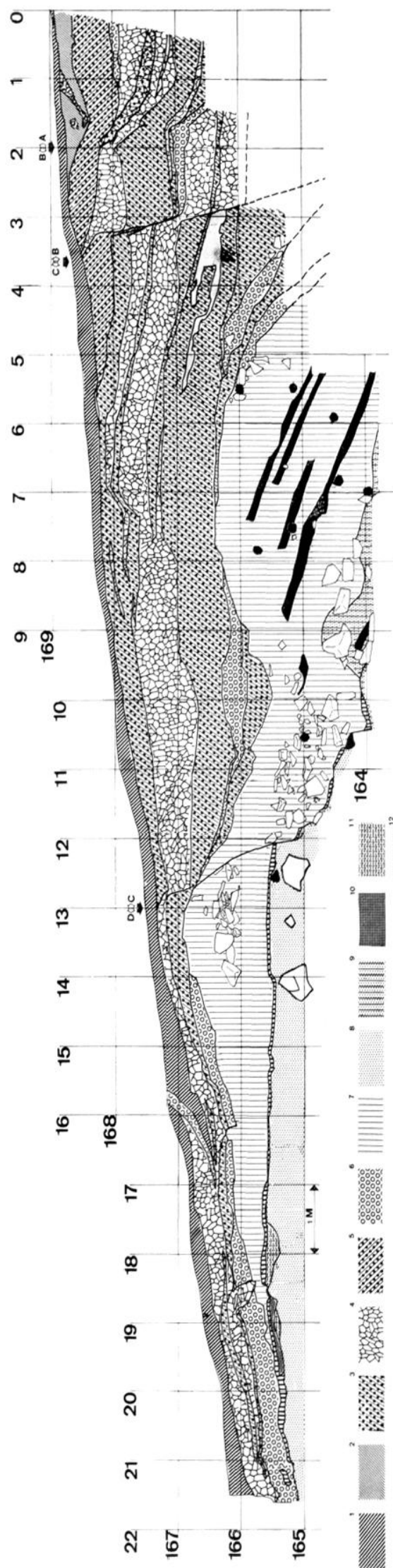

$\ddot{2}$

$\dot{\underline{\Sigma}}$

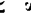

$\stackrel{\Xi}{\Xi}$

章苛

$\div=$

$\bar{\vdots}$

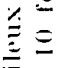

ㄱ.

$=2$

茜

$\therefore \frac{\pi}{\pi}$

$\ddot{2}=\hat{\Xi}$

就

플

$-$

范

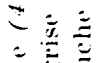

产

言

$\overline{-}$

$\because \because 2$

$\dddot{2}=$

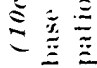

$\bar{\vdots}$

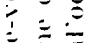

三”

产

$\frac{\bar{\pi}}{x} \bar{y}$

$\because \ddot{\partial} \equiv$

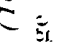

$\doteq$

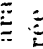

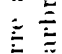

$\vdots$

$-\bar{\pi}$

$\Xi$

产

竞

$\equiv$

$\pm$

$\div$

$\vdots$

言紊

$\therefore=$

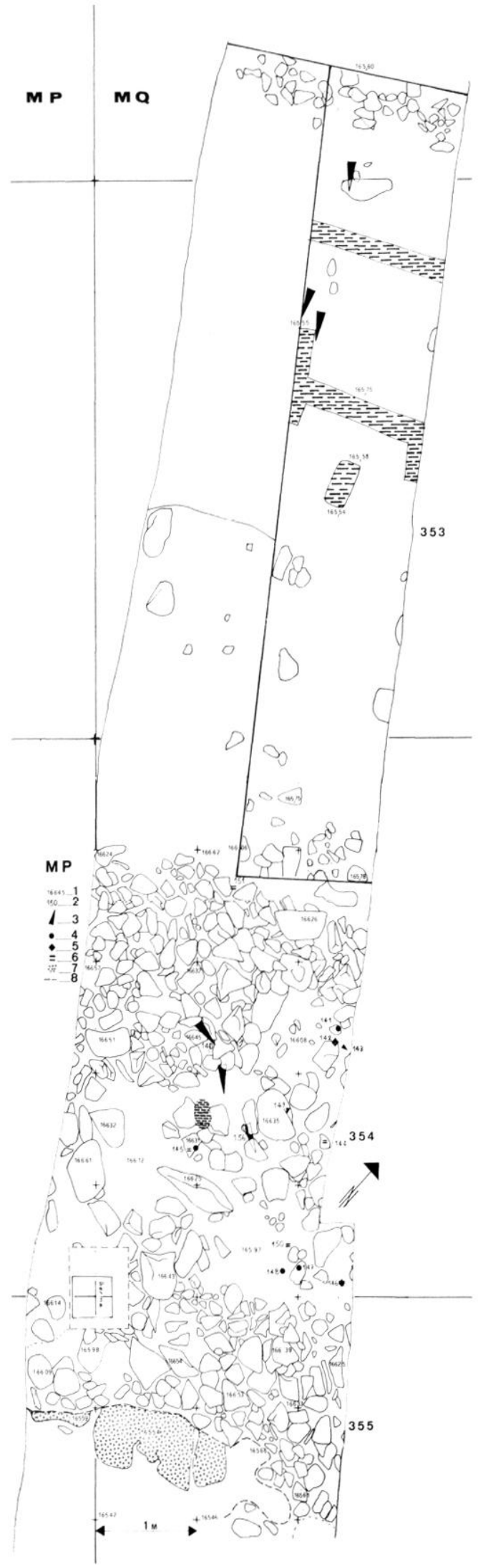

6 Plan de la tranchee $f$ : sommet du murus yallicus of iboulis du parement externe: 1 allitude N.G.F.;2n" d'objet ; 3 clou du murus gallicus ; 4 silex ; 5 ceramique: fi os ; 7 blocs de gries naturels en place; $x$ limites de lal rarriere morlerme. 
La couche sableuse rouge (3). qui se distingue de la précédente par sa couleur dominanle ot la presence de fragments de croute ferrugineuse tres dure est disposée comme une rlape sur le

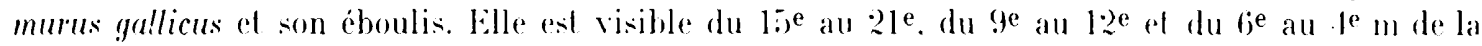
coupe : dans ce dernier secteur, elle se divise en plusieurs niveaux qui suivent exactement le pendage du parement interne : l'analyse du niveau 9 r. qui a mont ré que ce sable avait ále lré. vient confirmer une disposition intentionnelle. On trouve dans cetle courhe la meme quantite el la mime répartition de matériel que dans la rouche 4 : aes deux ensembles sont done parfaitement romparables. la courhe :) se distinguant seulement par une densité beauroup plus grande de mobilier.

La conche grise marbre de ronge (2) est divisée en plusieurs niveaux : 2 a siblend entre les deux parements du murus gallicus: 2 a apparait au surl du parement externe: 2. 2 e est un horizon plus sableux sous 2 a: 2f désigne un melange d'argile. de blocs de gres. de rharbon de bois recouvert par 2 e.

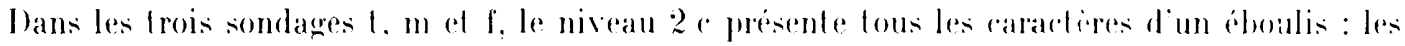
hlocs de grese reposent a sec les uns sur les autres, sams ordre : l'opaisseur de la rourbo diminue au fur et à mesure que l'on śéloigne du rempart vers le sud a la presence d'arqule grise enrobant les blors de gries et se glisiant parfois entre eux vient confirmer que 2 a appartient a la mème courhe que 2 a. Cette argile grise. qui ronstitue la rouche 2 a, est issue d'une région tris proche sur la colline. Elle s'étend du 5e au 12e m de la coupe sur une épaisseur moyenne de 2 m. Les horizons 2 a et 2 c contiennent un grand nombre de silex, alors que 2 e el $2 f$ en romportent tris peu. les ossements sont nombreux dans 2 c et 2 f. rares dans 2 a et 2 e. La céramique est roalement mieux représentée dans 2 ( el 2 f que dans 2 a el 2 e.

La couche de sable jaune (1) qui s'étend du $13^{\mathrm{e}}$ au $21^{\mathrm{e}} \mathrm{m}$ se divise on 2 niveaux : 1 a, sable jaune contenant des blocs de gres en formation : c'est la courhe géologique en place : 1 h s'otend au sommet de 1 a ; il s'en différencie par la presence de charbon de bois qui lui donne une teinte grise et contient de nombreux objets : ossements et tessons de ceramique sont parliculierement nombreux, comme dans la rouche $2 \mathrm{f}$.

Ies failles 1 ot 2 se manifestent par un décalage tres net observable dans les couches calcaires, et par deux lignes sombres visibles dans les couches sableuses inférieures. La déformation des couches au voisinage de la faille indique le sens des effondrements.

I a faille 3 est plus difficile à saisir, mais les preures de son existence se sont accumulées au cours de la fouille : elles nous ont amenés à admettre que le compartiment Ca s'elait effondré par rapport au compartiment l), alors qu une lecture rapide de la coupe pourrait, faire croire que la couche 1 a été coupée par la couche 2 a, comme pour ancrer une fondation. Mais trois observations confirment l'existener de la faille : la rourle caleaire du compartiment (s s'arrête brutalement et reprend jo cm plus haut dans le compartiment I) ; (ee phénomène a été observé sur les 7 coupes nord-sud éturliées dans les 3 sondages; la faille se prolonge au niveau de la limite entre les couches 2 e et 2 a. Nous avons vu plus haut que 2 c est l'éboulis de 2 a ; or cet eboulis se trouve placé plus haut que la structure dont il est issu : il faut en ronchure que 2 a sest affaissee. Nous observons en outre que de 16i7 à 166 n d'altitude, les pierres du parement sont fortement inclinés vers le nord ; rette disposition confirme l'hypothèse d'un aftaissement du rompartiment ci rar le parement. si grossier soit-il, n'aurait pas pu reposer sur des pierres disposeses de cette maniere.

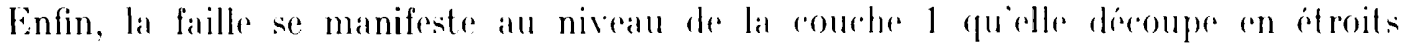
compartiments. L'analyse des horizons 1 b et ef revele une eomposition analogare ed caracteristique deune rourhe d'oreupation humaine : les ossements sont tres nombreux. le charbon apparait fréquemment sous forme de taches noires el donne a la terre une 

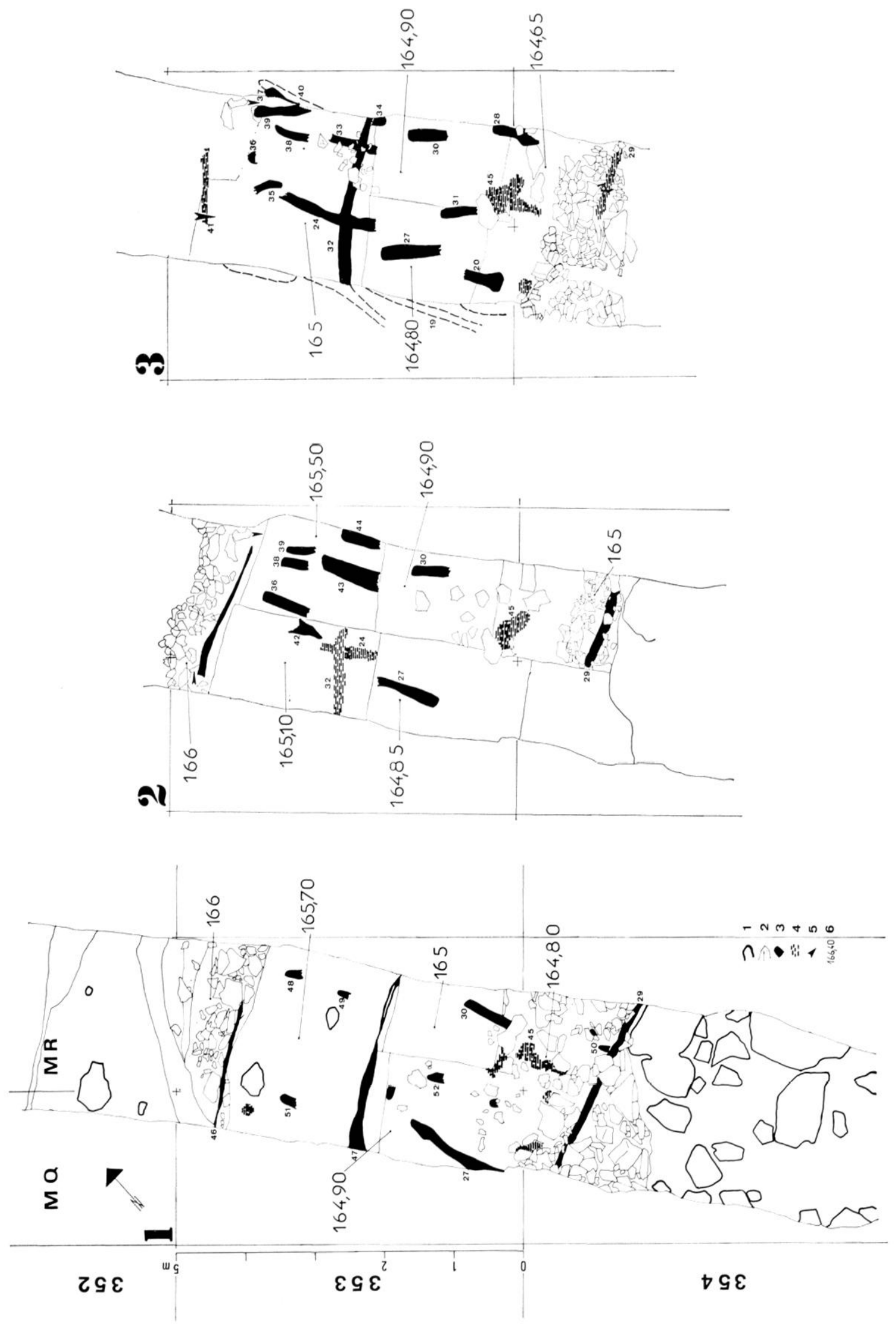


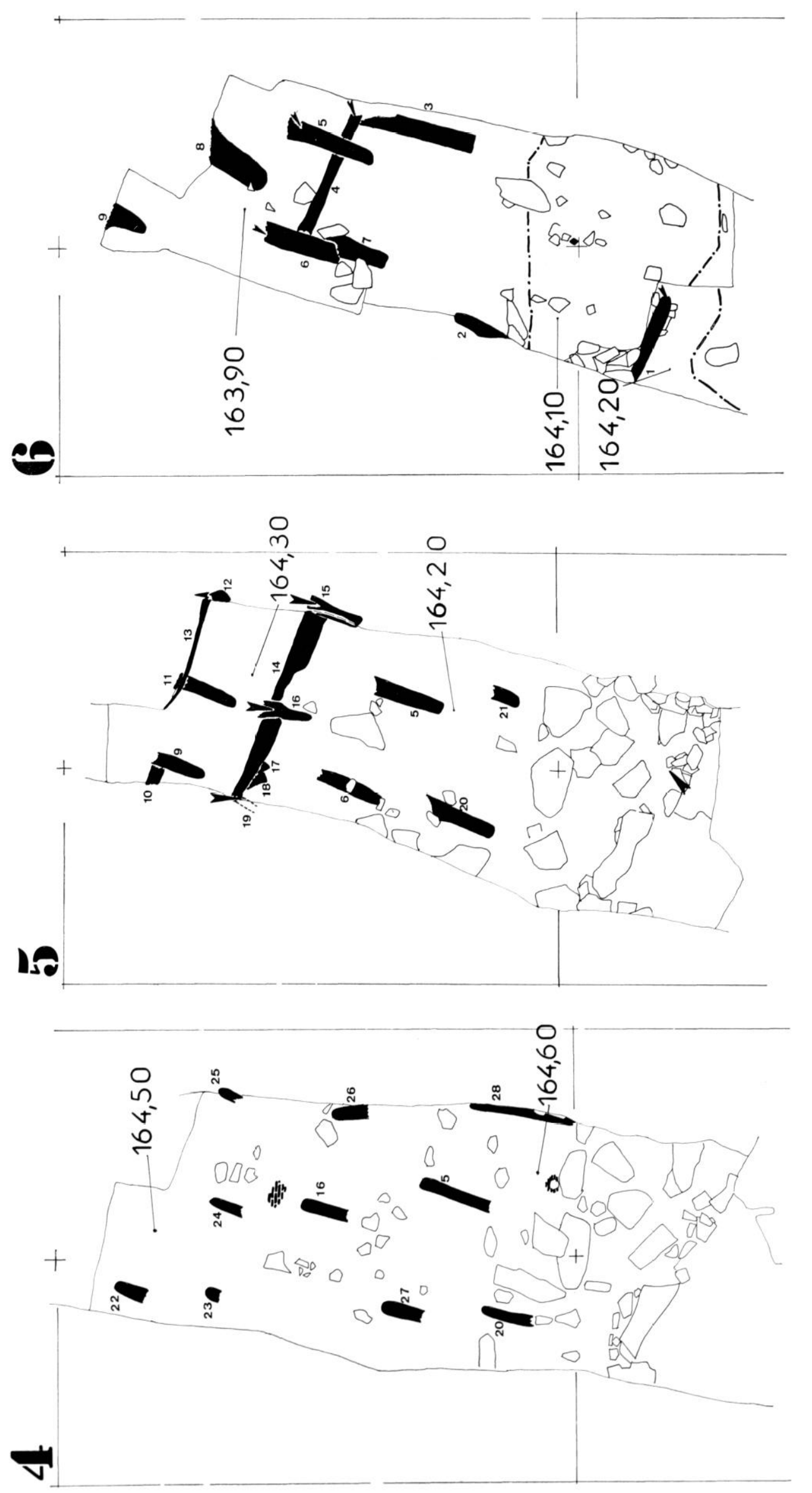


teinte grise. I)ans les tranchées f et $m$, alors que ces horizons ne représentent qu'un très faible volume (environ $1 \mathrm{~m}^{3}$ ), ils ont livé la moitié des ossements et plus du quart de la céramique. La couche 2 f est divisée en compartiments; le premier, large de $15 \mathrm{~cm}$, au $12 \mathrm{e} \mathrm{m}$; le deuxième, large d'1 m, du $12 \mathrm{e}$ a11 $11^{\mathrm{e}} \mathrm{m}$; le troisième, large de $1,50 \mathrm{~m}$, du $11^{\mathrm{e}}$ au $10^{\mathrm{e}} \mathrm{m}$ de la coupe. Ces compartiments sont placés à des altitudes différentes. Ils ne peuvent pas s'être déposés sous cette forme, car ils sont établis sur du sable. Il faut donc admettre que 1 b et 2 f sont un seul et même niveau d'occupation affecté par la faille 3 qui se divise à cet endroit pour former une série de petites failles annexes. Deux collages de tessons découverts dans la couche 1 b d'une part, dans la couche 2 f d'autre part, levent les derniers doutes.

Par rapport au murus gallicus, les objets découverts dans le niveau $2 \mathrm{f}$ seront plus anciens ; les objets découverts dans le niveau 1 b sous l'éboulis plus anciens ou contemporains; parmi les objets découverts plus au surl dans le niveau 1 b peuvent en outre se trouver des éléments postérieurs à l'écroulement du rempart.

On peut décomposer l'occupation du site en 5) phases:

1. Sur la couche de sable naturelle, qui semble aroir èté nivelée car elle présente une surface parfaitement horizontale en dépit de la pente du terrain, une occupation dense a entraîné le dépôt d'une couche épaisse de $10 \mathrm{~cm}$.

2. Ln murus gallicus est construit sur cette couche, peut-être nivelée écralement à rette occasion.

3. Le parement externe s'écroule partiellement sur la couche 1.

4. Les couches 3, 1 et 5 sont rapportées intentionnellement sur cet ensemble avant même qu'un sol ait eu le temps de se former sur le murus gallicus écroulé.

5. Lne série de failles affecte l'ensemble des couches ; dans la mesure où cet événement ne provoque pas de dislocation importante dans le quadrillage des poutres, on peut penser qu'il s'est produit avant leur pourrissement. J'érosion et les travaux agricoles tendent à régulariser la surface du sol et provoquent ainsi la disparition presque totale d'un niveau calcaire, conservé seulement dans les compartiments $\mathrm{A}$ at $\mathrm{B}$.

\section{LES STRUCTLRES}

1. La couche d'occupalion. La seule structure découverte à ce niveau est un petit foyer situé dans le carré 3\%) .MQ, dans la berme entre les tranchées f et m. La faible surface fouillée explique l'absence d'autres traces.

2. Le murus gallicus. Comme nous l'avons expliqué plus haut, des phénomènes géologiques ont affecté le rempart; c'est pourquoi nous ne pourons pas conclure sur certains détails de construction, cet inconvénient étant rattrappé par la conservation exceptionnelle de la fortification sur plus de $2 \mathrm{~m}$ de hauteur. Nous adopterons la forme de description utilisé par Mrs Cotton'.

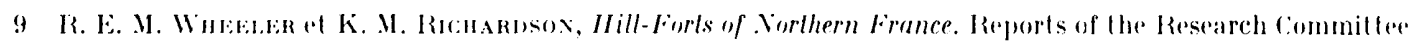
of the Society of Anlicpuries of London, XIX, 1957. Wilh an appendix on Nuri Gallici by .1. A. Collon. 

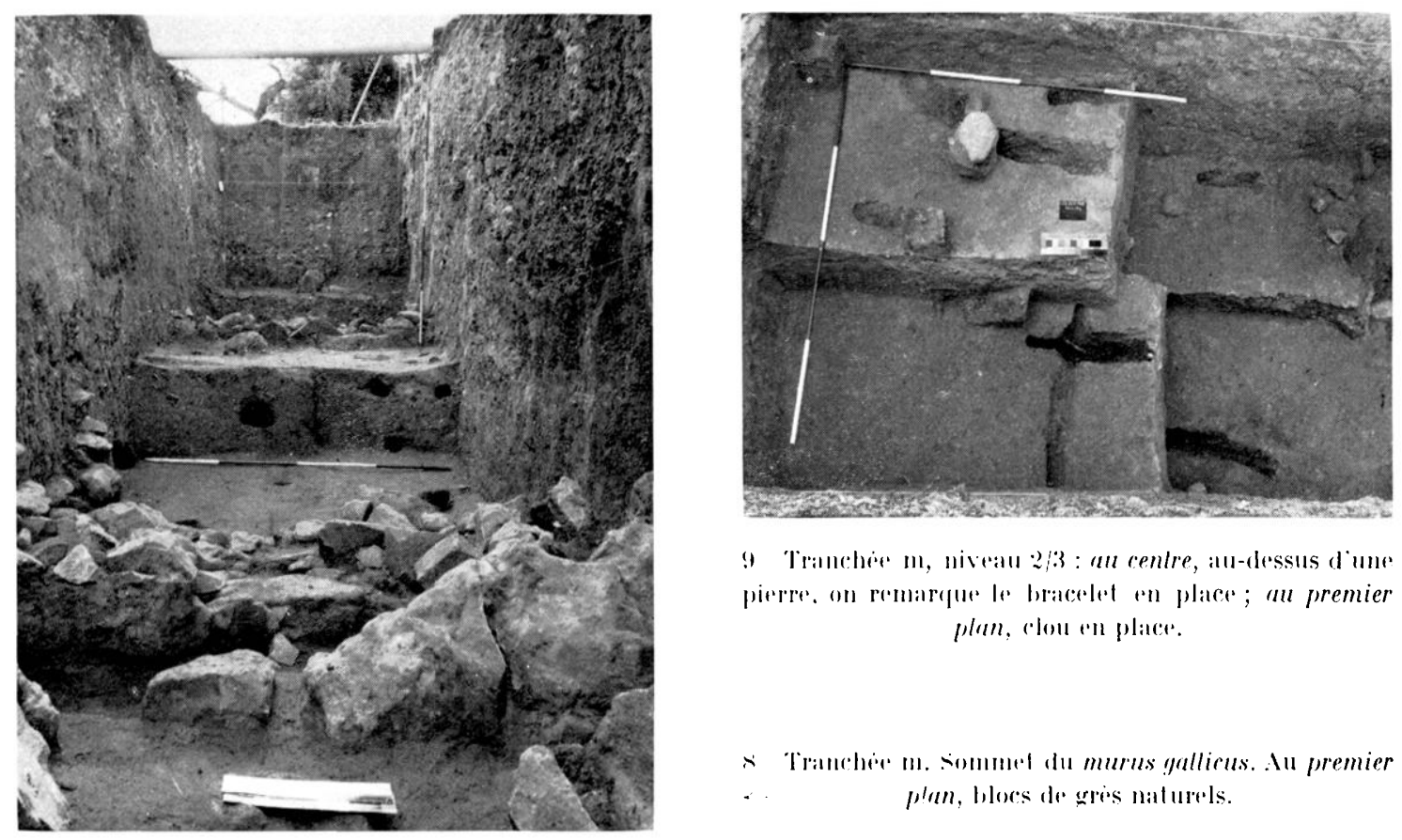

4 Tranchio m, niveau 2/3: an centre, au-dessus diune pierere, on remarque lo bracelet en place; an premier plan, clon an places.

* Trancher m. Sommef du murus gallicus. Au premier plath, hlocs do gres naturels.

Poutres transicersales (fir. 7 a 11) : nous ne pourons pas affirmer que le sol a été nivelé immédiatement arant la construetion du mur ; cependant on peut le supposer si l'on considere la couche d'oreupation qui est horizontale en avant du rempart. Le niveau $2 \mathrm{f}$ n'a été éturlié que sur $2 m^{2}$; sur cette étroite surfare, une poutere était en contact rirect avec la rourhe doceupation. Les intervalles entre les poutres transversales ne sont pas absolument constants : ils varient de 1 a $1,50 \mathrm{~m}$ et se trourent de ere fiat dans la moyenne observé sur les autres remparts de ce type. On pent observer, à la hauteur du 7 e m de la tranchée m. jo poutres transversales superposées : leur intervalle dans l'axe vertical est de 50 cm. Les poutres sont décalées dans le plan horizontal d'environ $50 \mathrm{~cm}$, conformément a la description de césar. Le sebouleversements qu a subis le rempart ne permettent pas de tirer de conclusions de linnelinaison des poutres. qui est actuellement de $200^{\circ}$. Nous n'arons pu constater aucune trace d'equarrissage sur les poutres transwersales; les empreintes laissées dans l'argile permettent de penser que les trones utilisées avaient un diametre très variable, te plus grand, exceptionnel, alteignant $20 \mathrm{~cm}$. La poutre observée sur la plus grande longueur mesure plus de $3,80 \mathrm{~m}$ (poutre 19). La poutre 50 pénètre dans le parement externe et vient se fixer à la poutre longitudinale :29.

Ies poutres diagonales sont totalement absentes.

Les poutres longitudinales se répartissent en trois sépies placeses respertivement dans le parement externe, dans le parement interne, et à la hauteur du ge m de la coupe, soit respectivement à 3.50 ed $1.70 \mathrm{~m}$ des parements externe et interne. La serie centrale comprend

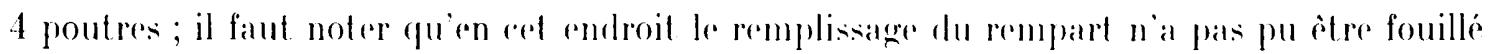

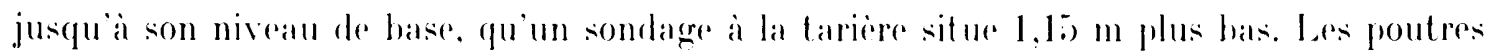

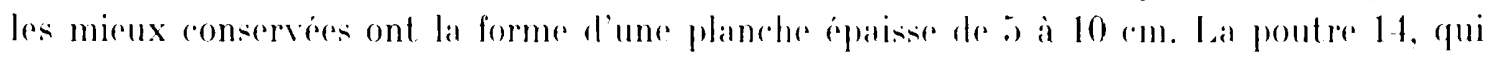

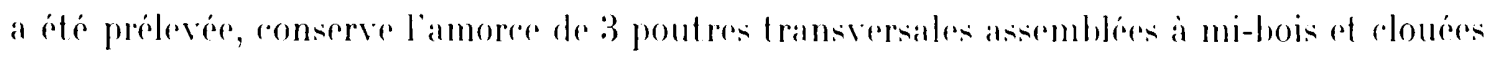




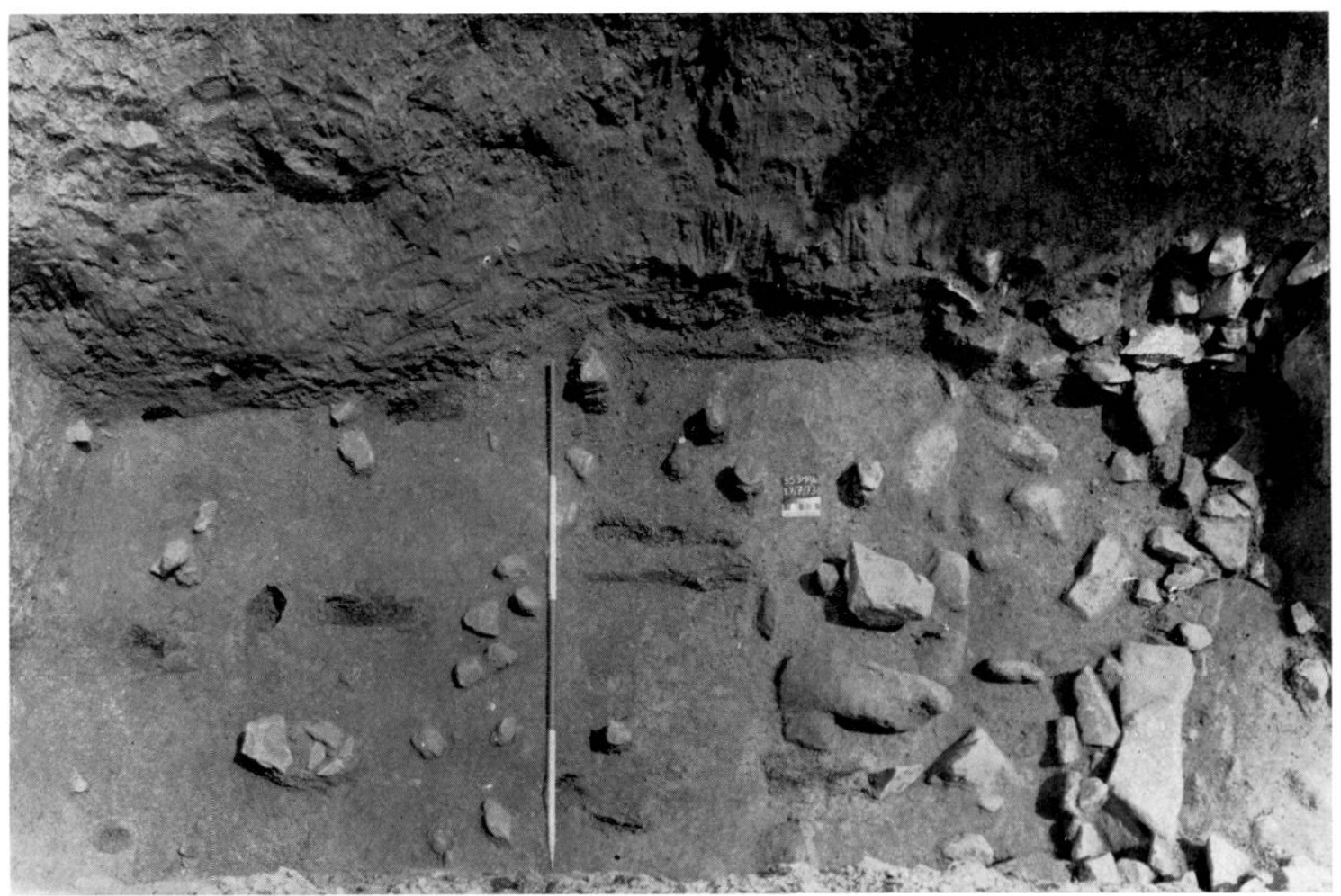

10 Tranchere m, nivean 4.

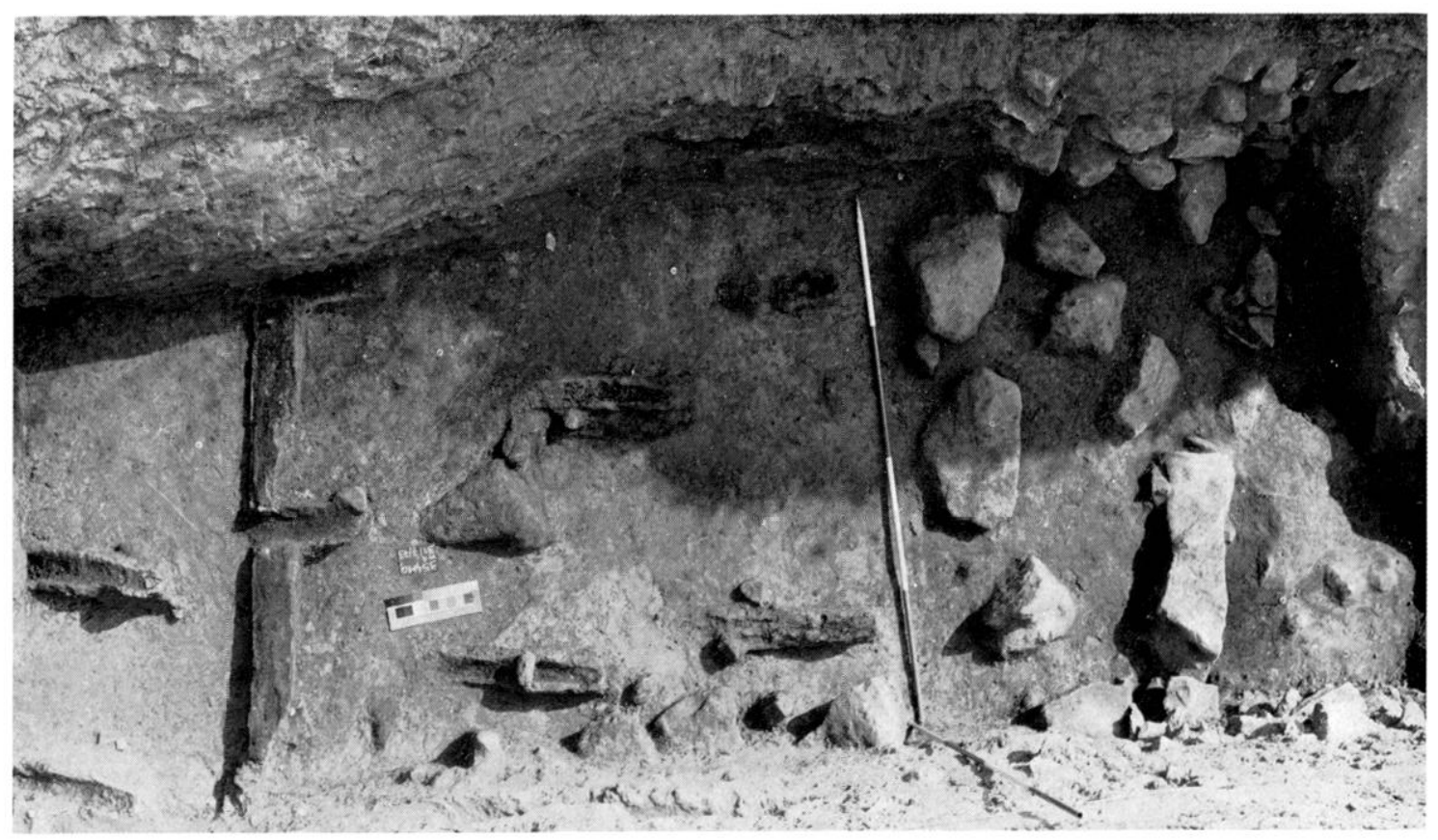

11 Tranchir m, nivialu 5. 


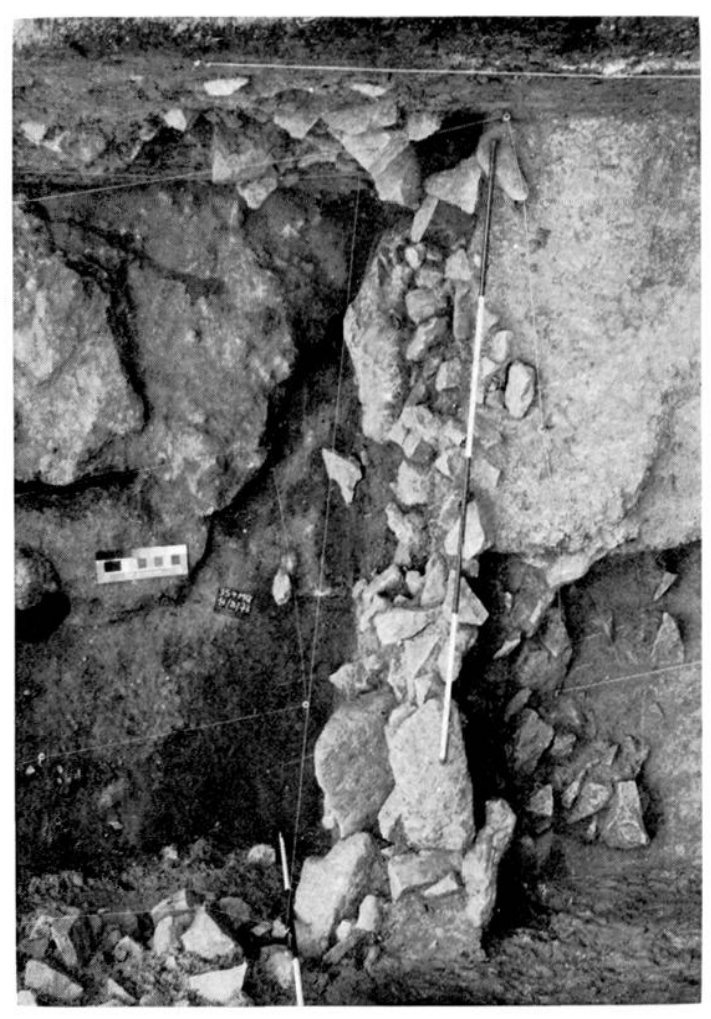

12 Tranchere f, sommel du parement externe. Son epaisseur est relalivement faible; il est composí de blocs de taille tres differente. Devant le parement apparaît une faille dans la couche 1.

13 Clous de la lanchere f. - ;

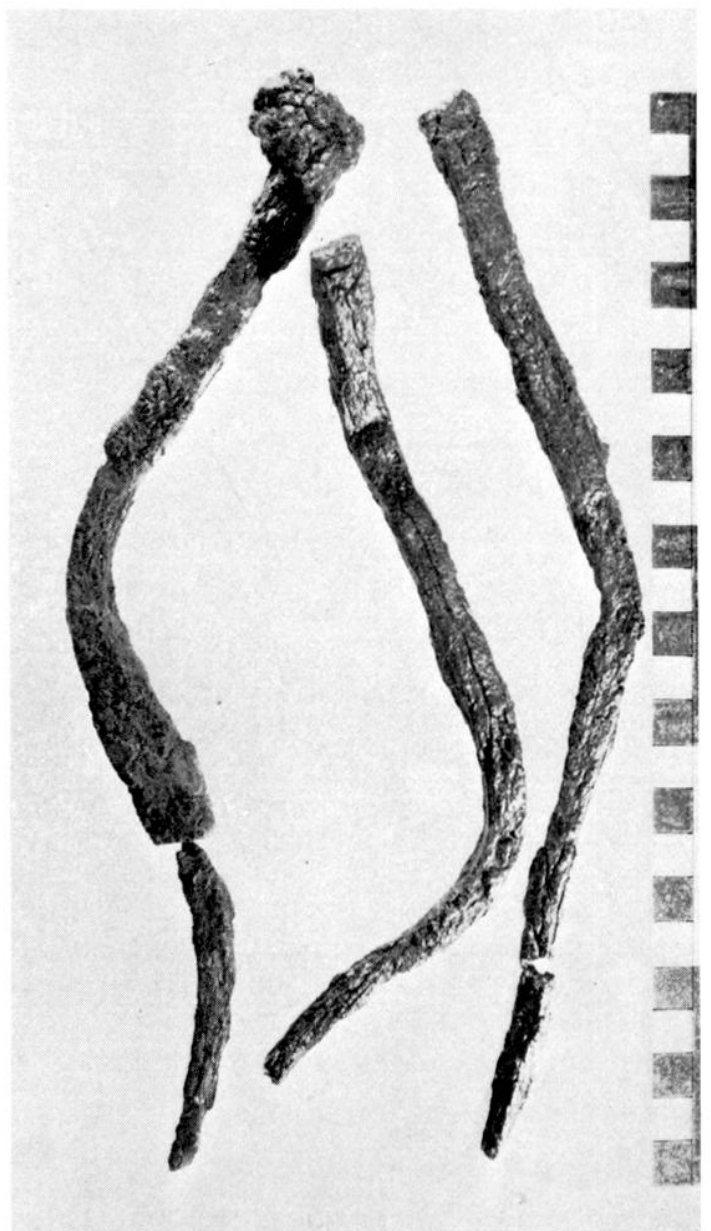

à $1 \mathrm{~m}$ les unes des autres; elle repose $50 \mathrm{~cm}$ plus haut que la poutre 4 . La longueur des poutres longiturlinales est supérieure à $2,50 \mathrm{~m}$, puisqu'elles traversent toute la tranchée.

Les (lous sont quardrangulaires, pointus, dépourvus de tête, et longs de 25 a a $30 \mathrm{~cm}$ (fig. 13). Ils servent exclusivement à la liaison des poutres longitudinales et transiersales.

Le parement externe a èté profondément affecté par la faille 3, ce qui rend la description difficile (fig. 12). Yous l'arons dégagé par niveaux successifs sur $2 \mathrm{~m}$ de hauteur. Il est composé de blocs de grès dont la taille, très variable, peut aller jusqu'à $50 \mathrm{~cm}$; ils sont visiblement choisis en fonction de la régularité de leurs cassures. Il s'agit d'un calage de pierres plutôt que d'un véritable mur, comme le laisse entendre César à propos du mur de Bourges : ... "grandibus in fronle saxis offarciuntur »... La limite entre le parement et le remplissage du rempart est difficile à déterminer, la largeur moyenne étant d'environ 1,50 m. La seule trace d'aménagement de la face externe du mur se reconnait à la disposition verticale d'une vingtaine de blocs. Bien que la présence de la carrière dans la tranchée f ait gêné le calcul du volume des pierres employées pour le parement externe, on peut estimer sa hauteur primitive à $3,50 \mathrm{~m}$ au minimum; ce chiffre est analogue à celui que donne Sir Ilortimer Wheeler pour Iluelgoat ${ }^{10}$. Yous n'avons pu déceler aucune trace de réfection du rempart. 
Le parement interne est un plan inclinè à 450. Il ne comporte des pierres qu'à son sommet sous la forme d'une chape large de $50 \mathrm{~cm}$ plantée dans l'argile du remplissage; ce sont des blocs de grès irréguliers dont la plus grande dimension n'excède pas $30 \mathrm{~cm}$. Catte chape sappuie sur la poutre longitudinale 46 sur laquelle sont plantés deux clous qui devaient la relier à deux poutres transversales disparues. On a observé sur d'autres sites la presence l'une rampe derrière le parement interne; la profondeur de la tranchée at la présence d'un chemin immédiatement au nord du sondage ne nous ont pas permis de vérifier son existence à levroux.

Le remplissage est composé d'une argile presque stérile comme au Mont Beuray; nous ne pouvons pas donner sal provenance exacte. Les quelques pierres éparses dans la masse du remplissage ne sont pas en relation avec les poutres, sauf peut-être dans le cas de la poutre 6. Sous avons observé. comme à Alésia, la présence de pierres posées obliquement derriere le parement externe en un point. Il n'y a pas de fossé, au moins dans les $12 \mathrm{~m}$ fouillés en avant du rempart; plus loin, aucune trace en surface ne permet d'en supposer l'existence.

3. Les conches supérieures. On a vu plus haut que ces couches avaient été déposées après l'écroulement du parement externe at avant la formation des failles, et qu'elles provenaient du sommet et de la base de la colline. Seule la couche 3 à l'endroit où elle recouvre le parement interne a pu être léposée au moment de la construction du rempart. Yous n'avons pas d'éléments chronologiques qui permettent de séparer les couches 4 et 5) : l'alternance des couches calcaires et sableuses incline à penser qu'elles ont été déposées ensemble. la témoin de la couche calcaire supérieure conservé dans les compartiments $A$ et $B$ permettent de supposer que Jes couches supérieures atteignaient $4 \mathrm{~m}$ d'épaisseur. L'apport d'une si grande masse de terre ne peut ètre motivé par des raisons agricoles, aucune technique d'amendement ne justifiant de dépôt d'une couche de calcaire epaisse d'un $m$. Il correspond par contre tout à fait à l'hypothese d'une reconstruction de la fortification : un rempart massif aurait succédé au murus gallicus. Il se serait élevé au-dessus du murus gallicus si la rouche de calcaire supérieure atteignait, comme la courhe inférieure, son point rulminant at son epaisseur maximum entre le $7^{\mathrm{e}}$ et le ge $\mathrm{m}$ de la coupe.

Le mat'́riei.

1. La céramique fig. 14 et 15 . Nous avons distingué 1.3 ratégories de céramique :

1. Céramique médievale ou moderne.

B. Amphores. Tous les fragments retrouvés peucent appartenir i des amphores républiraines: le seul col découverl est du type Dressel I ancien llivre iourte l ris écarté du col).

C. Briques et tuiles médiévales et modernes.

I). Tuiles ou carreaux antiques ou médiévaux.

I. Celle ratégorie comprend seulement 33 fragments issus d'un mime vase (pot ${ }^{11}$ tourné dans une paile blanche. couverte de mica et décorée a la moletle découverts a la limile entre les couches:3,

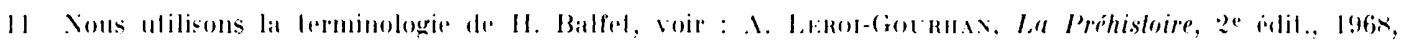
1. $2: 2 \cdot 2-2 \pi x$. 


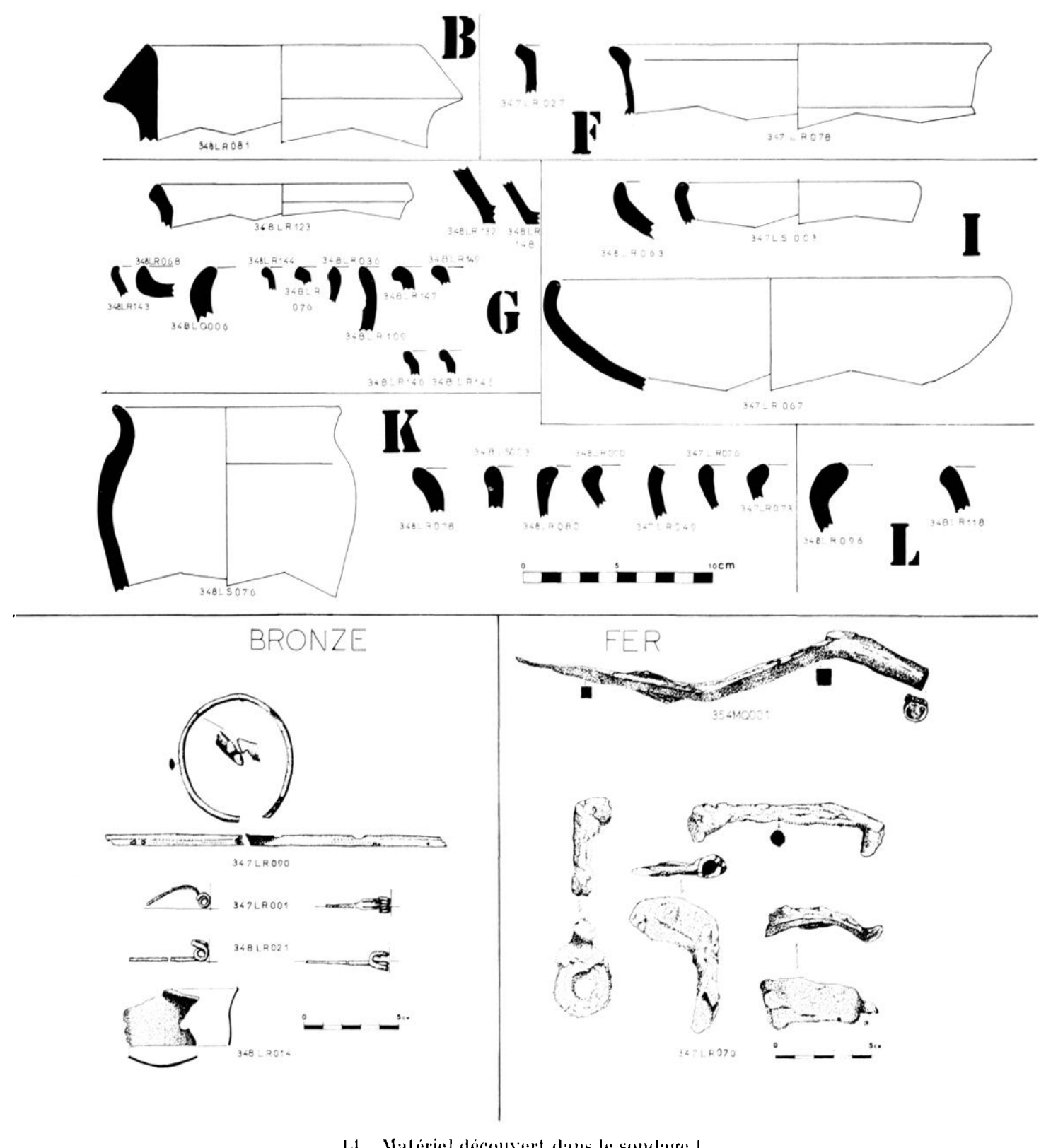

I. Vateriel decouvert dams la somdagel.

2 a et 2 r dans la tranchée f. Ils ont donc été déposés immédiatement apres l'éboulement du parement externe.

F. Céramique gris-noire tournée à la surface triss lisse. Parmi les formes, plusieurs pols et une terrine carenée. La forme 334 $\mathrm{XI}$ (2:37 correspond soil a un pelit bol, soil a la partie centrale du fond d'un vase.

(i. Céramique grise lisse lournée, représentée par de trés pelits fragments dont la plupart semble appartenir à des pots et trois seulement a des jalles. Deux tesons sont décorés. l'un d'une large baquette peu marquee. l'autre de traits incisés horizontaux.

II. Céramique tournée gris vert à lin dégraissant. La seule forme idenlifiable est une écuelle: un tesson est décoré d'une baguette. un autre de lignes horizontales pointillées en creux. 


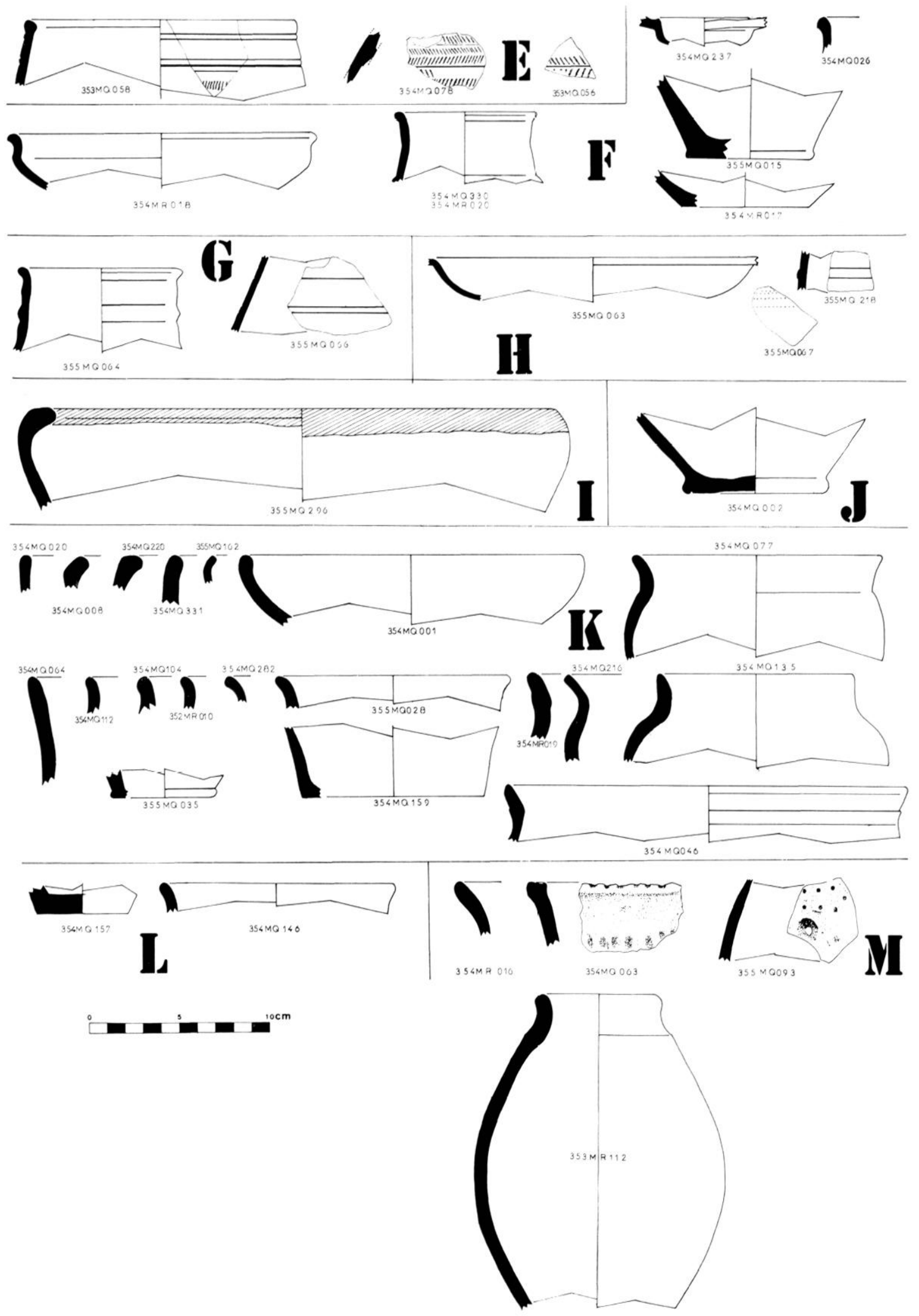

15 Ceramiques decouvertes dans les sondages f et $\mathrm{m}$. 
1. Céramique épaisse non lournée, de couleur rouge lissée, conlenant un dégraissant moyen; les formes sont des écuelles ou des bols au bord rentrant. Le bord du vase 3jis) II() 296 est peint en noir.

J. Céramique orange, probablement non tournée, à dégraissant moỵen composé de céramique pilée: la seule forme représentée est un fond.

K. Céramique mal ruite de couleur irréguliere variant du rouge au brun et au noir, celle derniere couleur dominant la série; non lournée elle contient un démraisint moyen ou grosier. La surface est peignée sur le corps du vase el lissée sur le col. Les formes sont des écuelles et des

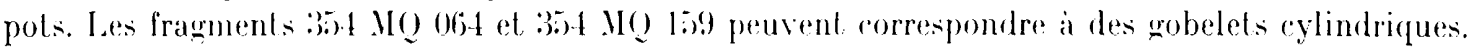

L. Cette catégorie ne se distingue de la précédente que par sa couleur dominante qui est le rouge.

II. Céramique trés mal cuite dont la paite est hétérogéne el le degraisisant grosier : il apparait a la surface des vases. la couleur est tres variable rouge brun. gris. noirt les parois des vases tres

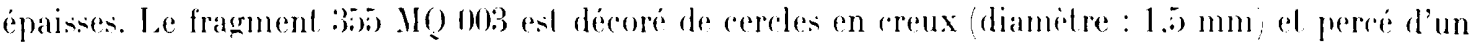

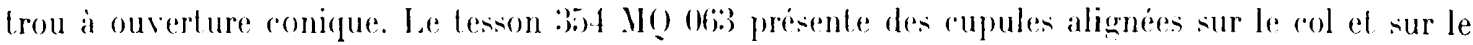
bord supérieur de la livre.

Le pourcentage de rhaque lype de céramique est sensiblement ident ique dans les deux sondages (voir tableau ri-dessousj. Les trois catégories de céramique non tournée ou simplement terminées au tour lent representent fo o du materiel. charune elant en quantile a peu pris égale. Ies amphores atteignent $10 \%$. la réramique toume $20 \%$. le solde comprend les éléments médiévaux ou modernes.

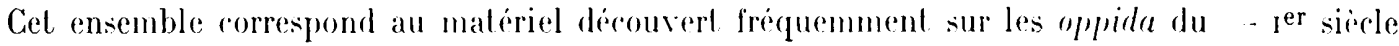
en tiaule cellique.

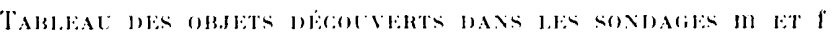

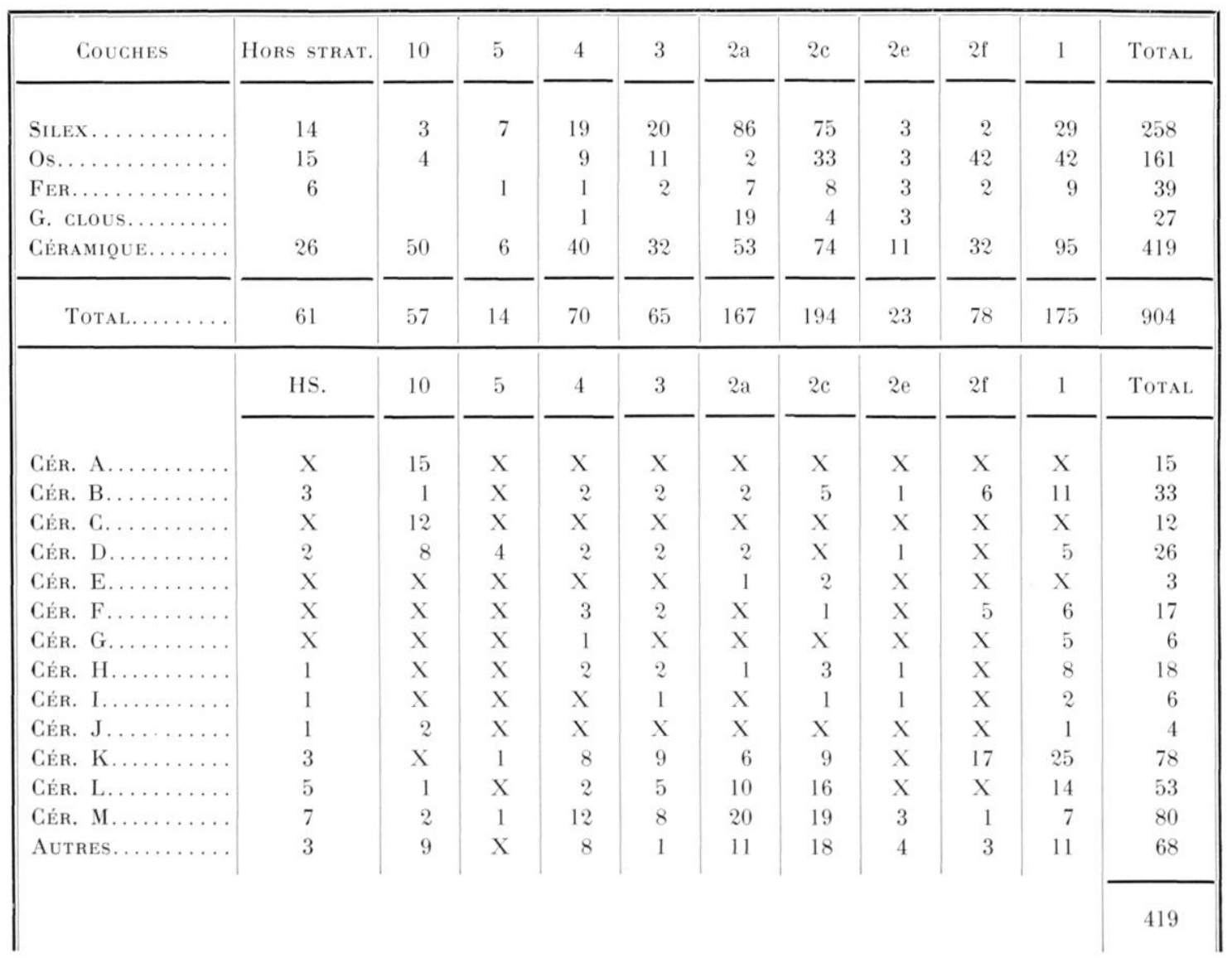


2. Le fer. kin dehors des clous du murus gallicus. les :3 sondages onl liver peu d'objels en fer. la seule trouvaille remarquable est l'ensemble de 30 objets 3.47 I.R 070 découvert dans la tranchée t fig. 14: sur la couche 1 a a s) (cm du parement externe, sous l'éboulis. Outre une vingtaine de clous, il comprend plusieurs barres de section carrée: l'une d'entre elles présente a chaque extrémité une protubérance: à cela s'ajoulent un anneau, un rochel et une serpe (?) a douille qui rappelle des objels découvert: a sainte-Blandine ${ }^{12}$.

3. Le bronze fig. 14 el 16 . Les différents sondages ont livé 3 libules : 347 I.R (0)1. dérivée du lype de Nauheim, double ressort a 4 spires. corde interne. L'arc plat est de forme générale triangulaire. mais un étrangle. ment marque le sommet. Il est décoré de 2 lignes incisées longiludinales. I e porte-ardillon a disparu. Cel objet provient de la Iranchée I ; il reposail au sommel de l'ebroulis.

348 I.R (221, ardillon et ressort double à 4 spires, corde interne; une des 4 spires el le reste de la fibule manquenl. Celle fibule a été découverte dans la tranchée 1, couche 1,
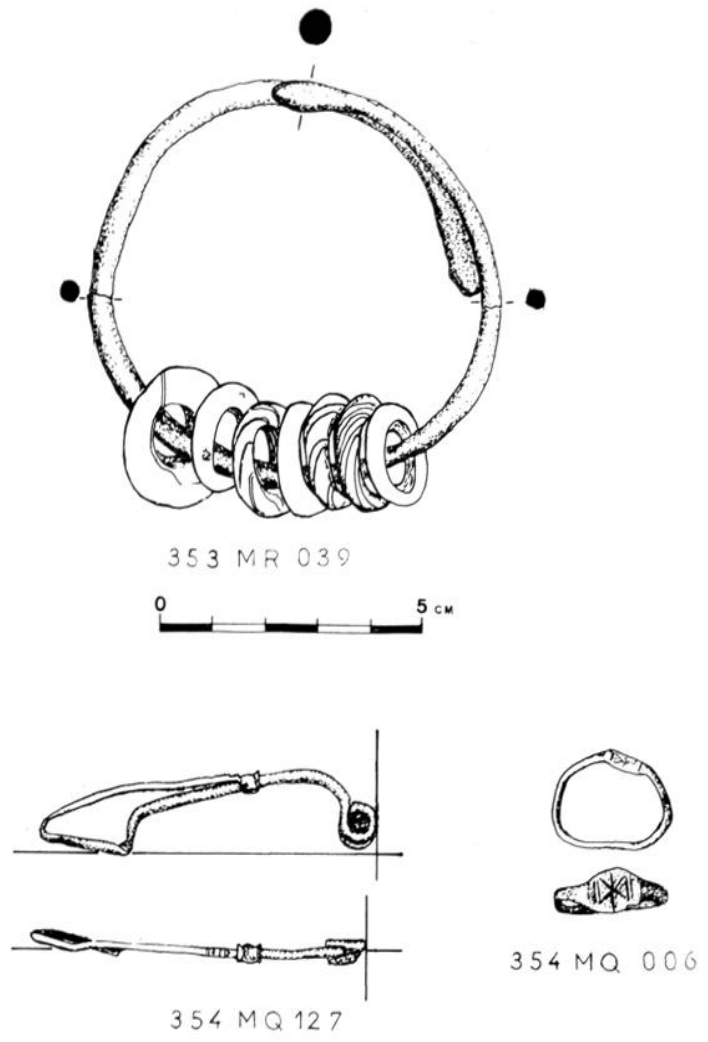

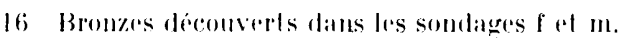
sous l'éboulis.

354 V( 127, fibule "pseudo-l a Tine 2 ", en fil de bronze, a ressort dont 2 spires sont conservés, enroulées aulour d'un axe en fer. L'appendice est replie sur l'are auquel il est fixé par martelage: 3 trails incises décorent son extrémile. Il manque une partie du ressort al l'ardillon. Cetle fibule vient de la tranché f. rouche 1, sous l'éboulis.

348 I.R 014, un frament d'objet rirculaire diamelre d'ouverture : 7 am . dont il formait. le bord, a été découvert dans la couche 1 . Iranchée 1.

lne bague en fil de bronze, aplatie en un point pour recevoir un molif de décor géométrique incisé a éte mise au jour dans la tranchée fouche 1 diametre environ : 16 mm

On voit sur la coupe de la tranchée I un bracelel forme d'un jone ovale (5) $\times 2.5$ mm; ouvert; la fermetures'effectue par une demi-queue d'aronde ; bien qu'il soit brise. on peul estimer le diametre à $6 \mathrm{~cm}$. Il est décoré de deux lignes pointillées en creux. I n bracelet analogue a été découvert sur la colline sainte- Blandine ${ }^{13}$.

La tranché a livé éxalement un bracelet en fil de bronze diamitre: 4 mm dont les exlrémités

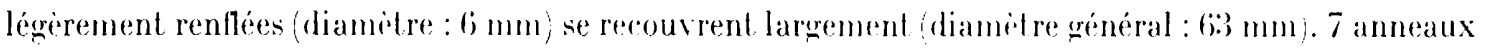
de verre sont passés autour de ce fil. II correspondent aux lypes 20 . 21 et 2:3 de T. E. Hacvernick ${ }^{14}$ et leur couleur est suceessivement : translucide avec paillon jaune. monochrome violet, translucide-brun avec filament en spirale jaune. bleu foncé, translucide brun avec filament blanc, I ranslucide -- brun avec filament jaune. bleu. De nombreux siles de la mîme époque ont livé des anneaux

12 G. Chaporat, Vienne Cianloise. le materiel de la Time III lromer sur la colline de Sainle-Blandine, Paris, ]970, pl. XV.

l:3 Op. cil., pl. II.

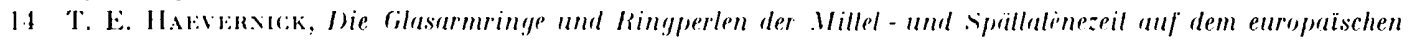
Festland, Bonn, 1960), R. (i. K., 1. XX.VI. 
identiques : Fort Harrouard, le Mont Beuvray, Sainte-Blandine, le Puy du Tour, etc. Plusieurs bracelets d'aspect semblable mais d'une taille nettement plus petite ont été découverts à Mathay (Doubs), dans un atelier de verrier, en association avec des fibules de Nauheim ${ }^{15}$. Ce bracelet est incontestablement contemporain de la construction du murus gallicus, puisqu'il repose sur la poutre 43 , au milicu du remplissage ; il est surprenant de constater que la seule monnaie trouvée dans le rempart (353 IIR 038) se trouvait à quelques centimetres de cet objet.

4. Les monnaies ${ }^{16}$. Nous avons découvert 4 monnaies en bronze :

348 LS 070, tranchée $t$, couche $1 \mathrm{~b}$, sous l'éboulis du parement externe. Monnaie des Leuci, tête ì grauche, B.N. 9147, L.T. XXXVII.

$348 \mathrm{LQ} \mathrm{001,} \mathrm{tranchée} t$, couche $1 \mathrm{~b}$, sous l'éboulis du parement externe. Monnaic à la tête de loup, pégase (?) à droite, Bituriges, B.N. $42 \ldots$

$3 \% 5 \mathrm{MQ}$ 173, tranchée $\mathrm{f}$, couche $1 \mathrm{~b}$, sous l'éboulis. Monnaie à la tête de loup à droite, pégase à droite au revers, Muret 94-95, anonyme des Bituriges.

353 MR 038, tranchée $\mathrm{m}$, dans le remplissage du murus gallicus; cette monnaie est presque fruste, probablement B... 6088, L.T. XIX.

Alors que cette dernière pièce a une diffusion assez large (Alésia, Pommiers, Vendeuil-Caply, Mont Beuvray, Titelberg, Saint-Marcel-Argentomagus), la précédente est. plus étroitement biturige (Bourges, Vendôme, Châteauroux, soings, Saint-Marcel-Argentomagus).

5. Le matériel osseux ${ }^{17}$. Les 160 ossements découverts appartiennent, comme nous l'avons $v u$, à la couche d'occupation. Le faible nombre d'éléments identifiables ne permet pas de tirer de leur étude des conclusions précises. Ils ne comportent aucun élément humain et reflètent une répartition entre les especces semblable à celle qui est observée sur les autres sondages ouverts à Levroux.

\section{LA DATATION}

La céramique comporte une proportion de vases en pâte grossière beaucoup plus forte que celle que nous avons observée dans les autres sondages ou au cours des prospections de surface. Tous les types rencontrés dans la fouille du rempart existent également dans le silo, la cabane de la Ruelle aux Roses et la cabane Lambert ${ }^{18}$, mais ils sont accompagnés de céramique du Cientre de la Gaule (céramique sigillée, cruches à pâte rose et engobe blane, gobelets du type Mont Beuvray, etc.) ou de sigillée d'Arezzo. Le lot découvert au rempart est plus proche des trouvailles de Vernou et la Rochecorbon ${ }^{19}$, qui comportent exclusivement de la céramique grossière et des amphores. Nous pouvons situer le rempart entre les autres découvertes de la colline des Tours de Levroux et ces deux derniers sites, puisqu'il comprend déjà un certain nombre de tessons tournés et plus fins. On peut provisoirement admettre l'hypothèse - toutes différences sociales mises à part —, que l'introduction progressive des techniques romaines se reflète dans la proportion céramique fine/céramique grossière, cette dernière devenant nettement minoritaire à Levroux à la fin du $\mathrm{I}^{\mathrm{er}}$ siècle av. J.-C.

15) IIusée des Antiquités nationales.

16 Fous remercions vivement le Dr Colbert de Beaulieu qui a bien voulu se charger de l'étude des monnaies.

17 L'étude des os a élé faile par .1 me .I. Derville qui prépare une maîtrise sur la faune gallo-romaine de Levroux.

Is Pour le silo, voir $\Lambda$ et 0 . Bcchsexscuetz, op. cit., p. 218 et s. Les autres chantiers sont en cours de fouille.

19 R. MAlGaro, Fouille de sauvelage dans le vallum d'un oppidum à Rochecorbon (37), dans Revue archéologique du Centre, XII, 1973, p. 187-208; pour Vernou, renseignements aimablement communiqués par M. J.-C. .Marquet. 
L'analyse de la céramique de la couche $2 \mathrm{f}$ ne permet pas de fixer avec précision un lerminus posl quem pour la construction du rempart, mais la présence des amphores dans cette couche la date au plus tôt du ir e siècle av. J.-C.

Le mobilier métallique dans son ensemble nous pousse à préférer dans cette fourchette une datation assez basse. Le bracelet 353 NR 037 et la monnaie 353 MR 038 sont strictement contemporains de la construction du rempart; la monnaie ne peut pas avoir été émise avant la guerre; nous avons vu d'autre part que le bracelet était caractéristique d'un milieu La Tène III assez avancé.

Le secteur de la couche 1 qui a été recouvert par l'éboulis du parement externe contient deux fibules et trois monnaies. Le fait que ces monnaies soient en bronze, et que l'une d'entre elles soit étrangère à la région indique, selon les observations des numismates, la fin de la guerre comme terminus anle quem non. Les fibules ne contredisent pas cette datation, sans toutefois apporter de preuves supplémentaires.

L'analyse du matériel montre, comme l'avait laissé prévoir l'étude stratigraphique, que l'ensemble des couches s'est déposé pendant une période assez courte : les couches 3 à 5 ne comportent, en effet, aucun élément plus récent que les niveaux inféricurs. La céramique de type E peut appartenir aux années -50 à $0^{20}$.

Place du Rempart de Levroux parmi les fortifications fon terre de la Tène III.

De multiples raisons poussent à choisir pour un site particulier un type de fortification de préférence à un autre : la présence de matériau adéquat, l'avancement des techniques de construction et de siège, l'urgence, etc. Dans leur étude sur les fortifications du Nord de la France, Sir Mortimer Wheeler et Katherine Richardson ont considéré que parmi les fortifications qu'ils avaient étudiées se dégageaient deux groupes en relation directe avec la guerre des Gaules : dans la région placée au nord-est de la Seine, des remparts en terre massifs précédés par un large fossé à fond plat, appelés type Fécamp par les auteurs. Dans la Gaule celtique, on a remarqué que les grands oppida étaient souvent défendus par un mur à parement externe vertical en pierre appuyé sur un poutrage de bois. Suivant l'hypothèse des auteurs, le murus gallicus était considéré comme "outside Belgic Gaul... suddenly and significantly to have become the standard anti-Roman device».

Dès 1958, Ch. Hawkes a contesté cette datation. Il pense, en effet, que ce type de fortification complexe ne peut se répandre que très difficilement dans une époque troublée. De plus il fait remarquer que Wheeler s'appuie sur le raisonnement d'E. Espérandieu qui date le murus gallicus de la fin de l'indépendance gauloise à cause notamment de son absence de la Gaule Narbonnaise. (Or, s'il n'existe aucun oppidum fortifié de cette manière avant la fin de La Tène II, aucune raison ne permet de lier à priori le développement des fortifications de la Gallia comala à celui des enceintes de la Narbonnaise, où l'utilisation du bois dans les remparts est rarement attestée.

20 Le laboratoire du Scottish Universities Research and Reactor Centre a effectué une datation par la méthode

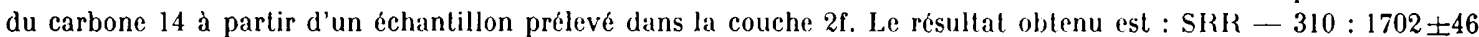
antérieur à 1950, soit $248 \pm 46$ ans de notre ère ; même corrigée par la dendrochronologie, celte datation reste très tardive, vers le $\mathbf{I I I}^{\mathbf{e}}$ siècle. Le matériel et les structures découvertes contredisent totalement cette datation. 
Bien que le murus gallicus date en général de la fin de l'indépendance, on peut citer plusieurs cas où les Gaulois, à une époque voisine de la guerre, ont choisi le rempart de type massif, vraisemblablement sans poutrage interne ${ }^{21}$. Bien plus, on connait plusieurs cas où le rempart massif vient se superposer à une fortification à parement vertical et, poutrage interne : c'est le cas à Châteaumeillant ${ }^{22}$, au Vieux Laon ${ }^{23}$. En Angleterre, Avery ${ }^{24}$ a décrit certains remparts sans structure interne nettement antérieurs au type Fécamp ; dans l'enceinte de Rainsborough (Northamptonshire), un rempart massif en terre est construit à la période IV sur un rempart à parement vertical partiellement écroulé qui lui sert de noyau. On a pensé que ce changement de technique pouvait être expliqué par la nécessité de reconstruire rapidement les fortifications : on peut citer plusieurs cas de défenses de type Fécamp dans le Berry, où l'on s'est battu dans les années - 52 et - 51 , à l'appui de cette hypothèse ${ }^{25}$; on rencontre le même phénomène de reconstruction dans le Sud-Est de l'Angleterre.

Si l'on accepte donc l'hypothèse que, dans certaines tribus, on a abandonné le rempart à parement externe vertical et poutrage parce que sa construction demandait trop de matériel et d'heures de travail, pourquoi a-t-on adopté comme solution de remplacement le rempart de type Fécamp? Selon les calculs d'Atkinson ${ }^{26}$, l'une des méthodes les plus efficaces pour construire un rempart massif conduit à éliminer la présence d'une berme entre le talus et le fossé et à minimiser la profondeur de ce dernier (on admet qu'il existe une profondeur minimum nécessaire pour l'efficacité de la défense), sa largeur étant déterminée par la quantité de terre dont on a besoin pour dresser le talus. Ce procédé semble correspondre au minimum d'effort, car le déplacement horizontal des matériaux exige beaucoup moins d'énergie que le déplacement vertical.

Il faut également considérer les techniques militaires de l'époque de César. Il nous dit, en effet, que le murus gallicus est un genre de défense très efficace contre le bélier et le feu ; mais le rempart en terre massif ne résiste-t-il pas encore mieux à ces techniques de siège? L'oppidum de Pommiers dans l'Aisne, défendu par un rempart et un fossé de type Fécamp, peut être identifié avec le Noviodunum des Suessiones, que César n'a pas

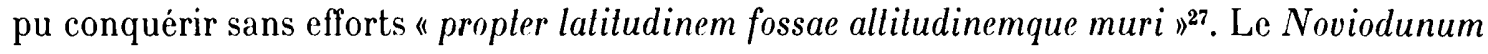
des Bituriges avait probablement des défenses analogues ${ }^{28}$. Il n'est cependant pas possible d'établir une relation directe entre l'introduction des machines de siège romaines et l'apparition du rempart massif ${ }^{29}$.

21 A. Ilogg et M. Cotton avaient déjà signalé la présence d'une part du rempart type Fécamp, d'autre part du murus gallicus, dans le département de la Vienne : A. H. A. HogG, A sample of French Irill-forts, dans Antiquily, XLIII, 1969, p. 260-273.

22 E. Hlgoniot et J. Gourvest, L'oppidum de Mediolanum, Châteaumeillant, Cher, dans Cellicum, I, 1961, p. 193-204.

23 G. Lossors, Les fouilles de l'oppidum gaulois du "Vieux Laon"à Saint-Thomas, Aisne, dans Cellicum, XV, 1965 , p. 1-26.

24 M. Avery, J. Sutrox et J. Baxks, Rainsborough, Northants., England: excavalions 1961-5, dans Proceedings of the Prehistoric Sociely, XXXIII, 1967, p. 207-306.

25 I. Ralston el O. Buchsexschutz, Late Pre-Roman Iron Age Forls in Berry, dans Anliquity, xurx, 1975, p. $8-18$.

26 R. J. C. Atкixsox, Neolithic Engineering, dans Antiquily, XXXV, 1961, p. 292-299.

27 Ċ́sar, De Bello Gallico, livre II, chap. 12.

28 Op. cil., n. 27.

29 De B. G., II, 12. 
Mais là où l'on a pu mesurer la hauteur des murus gallicus, on a obtenu un résultat voisin de $3,50 \mathrm{~m}$ (Levroux, Huelgoat); Bulliot estime que celui de Bibracte atteignait $5 \mathrm{~m}$, qui reste une hauteur encore faible par rapport aux remparts de type Fécamp. A titre de comparaison, on remarque avec Winter ${ }^{30}$ que la construction de murs très élevés au milieu du premier millénaire avant J.-C. coïncide en Grèce avec l'introduction de machines de siège. Des considérations analogues ont donc pu favoriser sinon la genèse, du moins le développement du rempart massif en Gaule.

Mais dans une société où la guerre, même moins acharnée que la lutte contre les romains, semble avoir été endémique ${ }^{31}$, il n'est pas nécessaire de lier étroitement l'architecture de fortifications aux activités de César.

Dans l'état actuel des connaissances, nous pensons qu'il faut considérer le murus gallicus et le rempart de type Fécamp comme des fortifications largement contemporaines en Gaule, hors de la Provence; on constate une tendance au remplacement du premier par le second.

Dans le cadre que nous venons d'esquisser, on peut situer la seconde fortification du site de Levroux par un rempart massif (probablement sans fossé à cause des matériaux utilisés) à une époque voisine de la conquête, ou légèrement postérieure ${ }^{32}$.

Olivier Buchenschetz et Ian 13. M. Ralston.

30 F. E. WINTER, Greek Forlifications, Toronto, 1971.

31 De B. G., V, 9.

32 Tant de personnes nous ont aidés pour cette fouille que nous craignons au moment d'adresser nos remerciements d'en oublier. Les propriétaires, MM. Triomphe, Franchet et Marchais ont eu la gentillesse de témoigner non seulement de la compréhension mais aussi de l'intérêt pour nos travaux. Plusieurs jeunes Levrousains, les équipes successives de stagiaires des Universités de Paris (Paris I, IV, VII, X) et le groupe archéologique de la Maison de la Jeunesse de Nanterre ont assuré une fouille assez austère avec beaucoup de ténacité. Nous remercions également Ch. Mennessier, A. el A. Schnapp qui nous ont aidé à l'encadrement. Anne Lecomte el Dominique Pette ont assuré les travaux de relevés et de dessins. Les travaux ont été financés par les Universités, la Maison de la Jeunesse de Nanterre, le Service des Fouilles et le Munro Fund de l'Université d'Edimbourg. 


\section{ANNEXE}

Observalions sédimentologiques sommaires (fig. 17 et 18).

Les couches du remplissage peuvent être rangées en quatre groupes suivant la part, prépondérante ou non, qu'onl pris les matériaux dont elles dérivent; ce sont, par ordre chronologique : 1, le calcaire Jurassique ; -..- 2, le sable Cénomanien ; - 3, les témoins du sol Éocène. - Il convient de rappeler que le substrat du coteau sous le rempart est Cénomanien et argilo-gréseux.
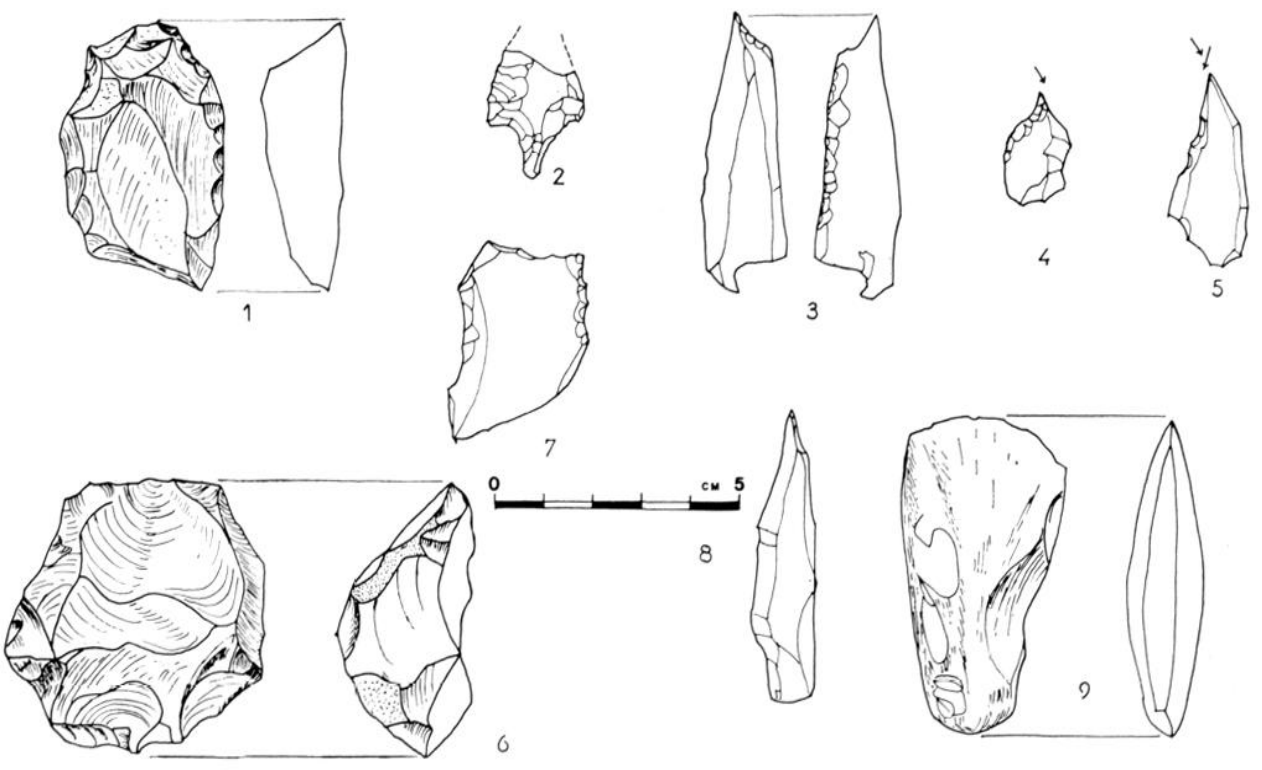

$\uparrow$

1733 outils et 200 éléments de debilage ont eté découverts principalement dans les couches de base, au voisinage du parement externe; ils s'apparentent aux silex d'une station proche, celle du Méz (dessin de M. Gratier).

18 Croquis de la coupe iludièe dans l'analyse géologique (tranchee m, paroi est, compartiments $\dot{A}$, B et $\mathrm{C}$ ).

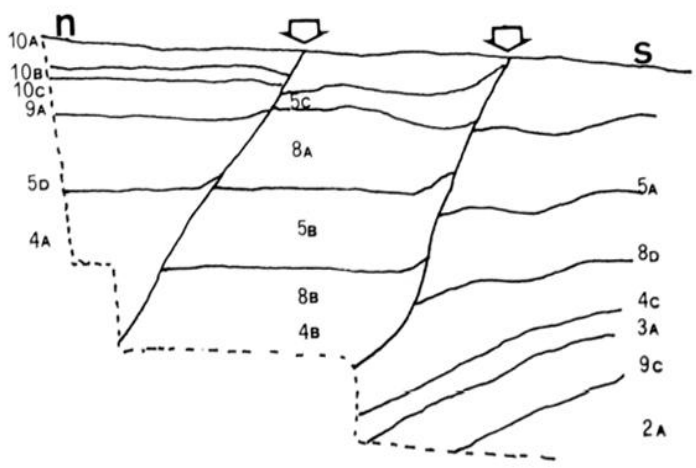

I. Groupe des couches dérivées du sol Éocène: nos 2 a, 9 c, 3 a. - Caractéristiques: texture argileuse avec sable grossier et quelques dragées de quartz; couleur bariolée : brun clair (7.5YR 5/8), brun rougeâtre $(2.5 \mathrm{YR} 4 / 6)$ à brun jaunàtre (10YR 6/8), gris jaunâtre (2.5Y 6/2) à jaune olive (5Y 5/3) ; présence caractéristique de fragments de croûte ferrugineuse noire à enduit argileux (couche 3 a). - Position: ces couches occupent la base de la stratigraphie, tapissant le Cénomanien. C'est une position normale puisque la pédogénèse tropicale éocène dont elles portent l'empreinte 
a affecté la partie superficielle du versant Cénomanien, dont elle reprend les matériaux. Le remaniement anthropique est donc limité en ce qui concerne ces couches, il s'est effectué sur place. Remarques: une recarbonatation éventuelle et limitée résulte d'une contamination par des éléments d'autres couches susjacentes, non par infiltration, mais par remaniement anthropique. La couche 2 a, qui s'étend sous une couche décarbonatée, en est un bon exemple. Lu couche $9 \mathrm{c}$ correspond à un sable probablement intentionnellement trié.

II. Groupe des couches dérivees du Cénomanien: $\mathrm{n}^{\mathrm{os}} 4 \mathrm{~b}, 4 \mathrm{c}, 9 \mathrm{~b}$. - Caractéristiques: sable fin micacé bien classé, friable ; jaunâtre pâle $(5 Y 5 / 3)$ à jaune olive $(2.5 \mathrm{Y} 4 / 4)$, parfois calcarifère. Position: dans la tranche moyenne du remplissage; elles sont plus sableuses que le substratum local (argilo-grèseux) et de ce fait proviennent de plus haut sur le coteau. Comme elles ne sont pas directement sur la roche mère de même âge, un transport, quoique limité, est à envisager.

III. Groupe des couches dérivées du Jurassique: nos 5 abcd, 10 b. - Caractéristiques: cailloux et plaquettes calcaires empâtés d'argile. - Couleur: jaunâtre pâle (2.5Y $8 / 3)$ et gris jaunâtre (2.5Y 6/2). Structure polyédrique lorsqu'il y a suffisamment d'argile, elle-même peu calcarifère. --Origine: c'est un apport venu de plus bas. Le calcaire de Champagne affleure à la base du coteau.

IV. Groupe des couches résullanl de mélanges: Cénomanien et Éocène : $-9 \mathrm{a}, 8 \mathrm{c}, 10 \mathrm{c} . \cdots$ Cénomanien et Jurassique : $-4 \mathrm{a}, 8 \mathrm{~d}, 10 \mathrm{a}$. -- Mélange de tout : $-8 \mathrm{a}, 8 \mathrm{~b}$. -- Remarques: $\dot{\mathrm{a}}$ l'examen morphoscopique, les quartz de la couche $8 \mathrm{c}$ se relèvent rosis et craquelés par l'action du feu : c'est pour l'instant la seule couche où l'on ait observé ce phénomène.

Rapporls des couches enlre elles: la couche $8 \mathrm{~b}$ peut résulter du mélange entre $5 \mathrm{~b}, 8 \mathrm{c}$ et $8 \mathrm{~d}$ : 4 a de $9 \mathrm{~b}$ avec $5 \mathrm{~d}$. La partie comprise entre les deux plans de discontinuité (compartiment $\mathrm{B}$ ) ressemble à un eflondrement par paquets à contre-pente; ceci est souligné par une structure feuilletée des couches suffisamment plastiques qui a détruit la structure préexistante le long des failles.

Michel Gratien ${ }^{33}$.

N.B.: La couleur des sédiments a été donné d'après les normes internationales (Standard soil color charts).

Détermination de traces de bois adhérentes à un clou du murus gallicus.

En plusieurs endroits, des traces appartenant i du bois sont observables : fibres ou fragments plus importants englohés dans la rouille du clou. Malheureusement, il a été impossible de déterminer s'il s'agit de bois feuillu ou de bois de conifère. Cn seul élément : la hauteur des rayons observés pourrait inciter à penser qu'il s'agit des gros rayons du chêne, dont la hauteur est habituellement importante. Cependant trop d'éléments manquent pour tirer une conclusion valable.

Cientre Technique du Bois.

Le chef du laboraloire d'anatomie, Y. TRENard.

Le chef du service commun des recherches el essais, A. VILLİ̈R:.

33 Pour replacer cette analyse dans son cadre, on se reportera à M. Gratier, Contribulion à l'élude géologique et préhislorique de la région de Levroux, U'niversités de Poitiers et Paris 1, A.D.E.L., 1974, 33 p. 